SUNY-NTG-92-4

\title{
The Propagation of Quarks in the Spatial Direction in Hot QCD $\mathrm{Q}$
}

\author{
V. Kochß, E.V. Shuryak, G.E. Brown, and A. D. Jackson \\ Department of Physics \\ State University of New York at Stony Brook \\ Stony Brook, NY 11794
}

\footnotetext{
${ }^{1}$ Supported by the U.S. Dept. of Energy Grant No. DE-FG02-88ER40388

${ }^{2}$ Supported by Deutsche Forschungsgemeinschaft DFG
} 


\begin{abstract}
The dynamics of light fermions propagating in a spatial direction at high temperatures can be described effectively by a two-dimensional Schrödinger equation with heavy effective mass $m_{\mathrm{eff}}=\pi T$. Starting from QED, we discuss the transition from three- to twodimensional positronium discussing the latter in detail including relativistic effects. In the case of QCD the problem is similar to that of heavy quarkonium. Our effective potential contains the usual Coulomb and confining parts as well as a perturbative spinspin interaction. The resulting $\bar{q} q$ "wave functions" reproduce recent lattice data for the $\rho$ and $\pi$ channels. The physical meaning of such 'confinement' is related to the non-trivial magnetic interaction of color currents in the quark-gluon plasma. Our results do not contradict the idea that the normal electric interaction of color charges is screened and produces no bound states in the usual sense.
\end{abstract}




\section{Introduction}

It is generally believed that hadronic matter at high temperature undergoes a transition to a new phase, the so called quark-gluon plasma (QGP), which is a gas of weakly interacting quarks and gluons. As far as global properties (e.g., thermodynamic properties) are concerned, this statement is supported by both perturbative QCD analyses and numerical simulations based on Lattice Gauge Theory (LGT). (See, e.g., general reviews [1], [2]).) However, this does not imply the absence of non-trivial phenomena even at very high $T$. As an example, one may mention the problem of magnetic screening, the mechanism for which remains unknown.

Some time ago, DeTar [3, 4] suggested that the QGP may be 'dynamically confined', in the sense that only color-singlet modes produce poles and branch points in linear response functions. In other words, he proposed that color-singlet modes might control the large-distance behavior of plasma disturbances. DeTar and Kogut [5, 6] pursued this matter numerically by calculating the correlation functions of various operators using LGT. The 'technical' point of importance for the interpretation of these results is that the correlations were measured in the spatial direction.

These functions were found to decay exponentially with distance, $\sim \exp (-M z)$, and DeTar and Kogut [5, 6] studied the temperature dependence of the exponent, $M$, (known as the screening mass) in a number of channels. The screening masses of the chiral partners $(\pi, \sigma),\left(\rho, A_{1}\right)$ and $\left(N\left(\frac{1}{2}+\right), N\left(\frac{1}{2}-\right)\right)$ were found to become equal within the accuracy of the calculations at the temperature where chiral symmetry is restored (defined as the point where $<\bar{q} q>\rightarrow 0$ and referred to below as $\left.T_{c}=T_{\chi S R}\right)$.

For spin zero channels, $(\pi, \sigma)$, and in the chiral limit, one would expect the mass to 
remain small even above $T_{c}$. These modes can be viewed as long-range fluctuations in the order parameter of the second-order chiral restoration transition as first proposed by Hatsuda and Kunihiro [8, 9, 10]. Indeed, lattice data show that $M_{\pi}\left(=M_{\sigma}\right)$ remains relatively small until rather large $T$. (Further discussion of this point and a compilation of data can be found in [1].). In contrast to this result, Eletskii and Ioffe [12 noted that for the other doublets the following is true: $M_{\rho}=M_{A_{1}} \simeq 2 \pi T=2 \omega_{0}$. For the baryonic doublet the mass is about $3 \pi T=3 \omega_{0}$. These values are essentially those suggested by the lowest quark Matsubara frequency, $\omega_{0}=\pi T$, and led to the suggestion that these correlators can be interpreted as describing the independent motion of two (or three) quarks in a plasma described as an ideal quark gas.

For people not comfortable with the Matsubara formalism, one can explain this point in a different way by looking at equal time correlations, as recently suggested by one of us [13. One can show that the propagator for free massless quarks at temperature, $T$, over a spatial distance $x$ in zero time is given by

$$
S_{T}(x)=\left(\gamma_{x} \partial_{x}\right) \int \frac{d^{3} k}{(2 \pi)^{3} k} \exp (i \vec{k} \cdot \vec{x})\left[\frac{1}{2}-\frac{1}{1+\exp (k / T)}\right]
$$

The last term contains Fermi occupation numbers, and the combination $\left(1 / 2-n_{F}(k)\right)$ is clearly related to the familiar combination $\left(1 / 2+n_{B}(k)\right)$ in the case of Bose statistics. In both cases the ' $1 / 2$ ' term is due to zero-point oscillations and the last term to the contribution of thermal excitations. However, quarks with small momentum have $n_{f}=$ $1 / 2$. Thus, one has a cancelation of these two contributions for $k \ll T$ which eliminates power contributions and leads to $S_{T}(z) \sim \exp (-\pi T z)$. Another amusing way to look at this comes by rewriting $\left(1 / 2-n_{F}(k)\right)$ as $(1 / 2)\left[\left(1-n_{F}\right)-n_{F}\right]$ so that the particle-hole symmetry $n_{F} \leftrightarrow\left(1-n_{F}\right)$ is made obvious. Since the situations near $n_{f}=0$ and $n_{f}=1$ 
are physically similar (up to the sign of the propagator), it is natural to expect that the midpoint, $n_{F}=1 / 2$, is special. There, the particle and hole terms cancel.

It is useful to mention that there are essentially two simple ways to make the connection between the imaginary-time formalism and real time. One is to look at equal time correlations (i.e., correlators integrated over frequency) as in the previous illustration. The other possibility is to study correlators at zero frequency (i.e., correlators integrated over imaginary time.) It is the latter which is used to extract the screening mass in LGT calculations while the former is used to determine the wave functions.

The scenario of free motion in a ideal quark gas is too simplistic to describe the real situation, but the observation that most screening masses are consistent with this picture suggests that other mechanisms have little net effect. In particular, these data provide an important limitation on any dynamically generated quark mass, $m_{d y n}$ [14. The screening mass for massive quarks would be $M=\sqrt{\omega_{0}^{2}+m_{d y n}^{2}}$. Thus, it seems that an appreciable $m_{d y n}$ is excluded even in the region $T \sim T_{\chi S R}$. A picture in which hadrons go massless 15] as $T \rightarrow T_{\chi S R}$ (leading to the scenario suggested in [16, 14]) is not excluded. (However, given a temperature of $T \simeq 150 \mathrm{MeV}$, an accuracy of about $5 \%$ for the calculated screening masses is required to rule out dynamical quark masses smaller than $m_{d y n}=150 \mathrm{MeV}$.)

The next important step was made by recent lattice studies [17] which revealed that the $\bar{q} q$ pair, moving over large distances in the spatial direction, is actually closely correlated in the transverse plane. Moreover, this correlation is even more pronounced at finite $T \simeq 150 \mathrm{MeV}$ than it is at $T=0$. It was suggested [17 that, in line with DeTar's dynamical confinement ideas, the quark and antiquark are not free at all but bound even at high $T$. The aim of the present paper is to provide a quantitative explanation of these data as well as a general discussion of what these data actually mean for the physics of 
the QGP.

An essential technical point, important for the understanding of the terminology used, is the interchange of the time and $z$-axis which we shall describe in some detail in the next section. After transformation to this 'funny space', one deals with a system at zero temperature but placed in a box periodic in the $z$-direction with periodicity $\beta=$ $1 / T$. Using this language it is obvious that, in the high temperature limit, a so-called dimensional reduction takes place. The $(3+1)$-dimensional gauge theory becomes a $(2+1)$-dimensional theory as suggested long ago 18. The main physical point is that, in the high $T$ limit, the motion of a quark in the 'funny space' is dominated by its momentum in the $z$-direction which is governed by $\pi T$ as a consequence of the antiperiodic boundary conditions. For motion in the transverse direction, as we shall show, this momentum behaves like a mass. Since at high temperature this 'mass' becomes very large, any attractive potential can bind quarks in the transverse direction.

The main objective of this work is to derive the high- $T$ limit of the "wave functions" introduced (and measured on the lattice) in [17]. In section 2 we start with the QED case and discuss how the transition from $d=3$ to $d=2$ positronium takes place. Qualitative features of $d=2$ bound states in the $d=2$ logarithmic Coulomb potential were recently discussed in ref. [19]. We report the results of a more quantitative analysis including nextorder corrections. We pay special attention to the spin-spin interaction because this issue is important for the mesonic splittings discussed in section 3 .

The effective $d=2$ QCD potential for a $\bar{q} q$-pair propagating in the spatial direction was studied on the lattice in ref.[20]. It was found to be essentially temperature independent. (This result stands in sharp contrast to the usual potential, for propagation in time, which exhibits both deconfinement and charge screening.) Although the data 
[20] are not very good at large separations and allow a wide range of interpretations, comparison with the "wave functions" allows us to conclude that the "string tension" is essentially $T$-independent in the interval between $T=0$ and $T=210 \mathrm{MeV}$ studied in ref. 177. Another issue addressed in section 3 deals with the differences, both in the shape of the "wave functions" and the screening masses, between the $\pi$ and $\rho$ systems. A spin-spin interaction of the Fermi-Breit form provides a natural explanation of most of these differences.

In section 4 we proceed to a discussion of the physical meaning of these "bound states". In order to understand these results, one must come back from the 'funny space' to ordinary space. One realizes that by dealing with static correlation functions (with no time involved), it is not possible to say anything about the energy spectrum of excitations in the QGP at high temperatures. What is actually studied is the momentum spectrum of the correlators for various channels. At $T=0$ the two spectra are identical. At high $T$, they are completely different. 


\section{Propagating an $e^{+} e^{-}$pair at high $\mathbf{T}$}

In this section we shall study the "wave function" of an $e^{+} e^{-}$pair at finite temperature in Euclidian space. We shall proceed by ignoring the Coulomb force and restricting our attention to the magnetic interaction between currents. The aim of this restriction is to preserve the analogy to QCD in which the electric interaction is screened and magnetic interactions are believed to be dominant at high temperature. The purpose of this example is that QED is an abelian gauge theory with a fixed and small coupling constant, e. One can work out all essential ingredients, and both the "binding energy" and the "wave function" of an $e^{+} e^{-}$pair at high $T$ can be obtained. As is well known, a quantum field theory at finite temperature, $T$, can be formulated using Euclidean time, $\tau$, ranging from 0 to $\beta=1 / T$ with periodic boundary conditions for bosons and antiperiodic boundary conditions for fermions. This formulation, which is the corner stone of LGT calculations, will be the starting point for our considerations. This convenient generalization of the $T=0$ case has, however, its limitations. One is unable to consider any time-dependent quantities and deals only with static (heat bath averaged) quantities. As a consequence, LGT cannot tell us anything about the frequency spectrum of elementary excitations. What we are going to study in this work is, therefore, related to the momentum spectrum. (In other words, properties related to spatial correlation functions.)

Given the magnetic nature of this problem, it is simpler to proceed by interchanging the (compressed) Euclidean time, $\tau$, and the spatial direction, $z$, in which the correlator is measured. This transformation leads us to a 'funny space' where the system propagates in unlimited time (hence, at zero temperature) but is confined in a box in the $z$-direction. This transformation must be applied to the fields. Specifically, the original magnetic field, 
$F_{13}$ and $F_{23}$, becomes an electric field (i.e., $F_{13} \Rightarrow F_{10}=E_{x}$ and $F_{23} \Rightarrow F_{20}=E_{y}$ ). The magnetic interaction between two rapidly moving charges is transformed into an electric interaction between two, nearly static, charges. We emphasize that this new Coulomb interaction has emerged from a technical manipulation. Its physical origin remains magnetic and, as such, it is not subject to the effects of screening which are presumed to suppress genuine electric interactions.

The presence of a spatially periodic "box" modifies the interaction of the charges. This modification is readily calculated for our simple example of small $\left(\alpha=e^{2} \ll 1\right)$ charges in QED with dynamics given by the current-current interaction of the leading onephoton exchange diagram. The $\tau z$-interchange immediately transforms this interaction into the ordinary Coulomb potential, $V=-\alpha / R$. The presence of the "box" boundaries modifies this result significantly because the electric potential must now satisfy periodic boundary conditions in the $z$-direction. There are two ways of solving the standard Poisson equation:

$$
-\nabla^{2} \Phi=\rho
$$

One can either (i) expand the field in Fourier components

$$
\begin{aligned}
\Phi(x, y, z) & =\sum_{n} \Phi_{n}(x, y) e^{-i 2 n \pi T z} \\
\rho(x, y, z) & =\sum_{n} \rho_{n}(x, y) e^{-i 2 n \pi T z}
\end{aligned}
$$

(in the high $\mathrm{T}$ limit only the $\mathrm{n}=0$ modes are important) or (ii) one can construct a periodic array of "reflected charges". (See Figure 1.) The second approach is certainly simpler; it involves no integrals and leads directly to the sum

$$
\Phi=\frac{e}{4 \pi}\left[\frac{1}{r}-2 \sum_{n=1}^{\infty}\left(\frac{1}{\sqrt{x^{2}+y^{2}+(n \beta-z)^{2}}}-\frac{1}{n \beta}\right)\right] .
$$


We note that the sum is automatically regularized at large $n$ and that the large $-T$ limit of this three-dimensional problem is simply the two-dimensional Coulomb potential:

$$
\Phi_{0}\left(r_{\perp}\right)=-\frac{e}{2 \pi \beta} \ln \left(\frac{r_{\perp}}{2 \beta}\right)
$$

which is equivalent to the potential of a uniformly charged wire (with charge density $e / \beta$ ) in three dimensions.

The fermion field is also affected by the $\tau z$-interchange. This is most easily appreciated by considering the Dirac equation for a single, massless particle in Euclidean space which has the form:

$$
\gamma_{\mu}^{E} \partial_{\mu} \psi=0
$$

where the $\gamma_{\mu}^{E}$ are Euclidean gamma matrices. The solutions to this equation are

$$
\psi(x)=\psi_{0} e^{-i(2 n+1) \pi T x_{0}} e^{-i \vec{p} \cdot \vec{x}}
$$

where the dependence on the 0-direction is due to the antiperiodic boundary conditions which result from the finite temperature. (Here, $\psi_{0}$ is a spinor which will not concern us.) The Euclidean Dirac equation for this problem is evidently invariant under the $\tau z-$ interchange, and all effects are concentrated on the wave function, eqn.(6), which becomes

$$
\psi(x)=\psi_{0} e^{-i(2 n+1) \pi T z} e^{-i p_{0} x_{0}} e^{-i \vec{p}_{\perp} \cdot \vec{x}_{\perp}}
$$

because of the boundary conditions. The Dirac equation is solved by the choice

$$
p_{0}= \pm i \sqrt{p_{z}^{2}+p_{\perp}^{2}}= \pm i \sqrt{((2 n+1) \pi T)^{2}+p_{\perp}^{2}} \equiv-i E
$$

with $E$ real. The wave function thus takes the form

$$
\psi(x)=\psi_{0} e^{-E x_{0}} e^{-i(2 n+1) \pi T z} e^{-i \vec{\perp} \cdot \overrightarrow{x_{\perp}}}
$$


which is the solution of a Dirac equation for a particle with a given momentum, $p_{z}=$ $(2 n+1) \pi T$, in the $z$-direction. For large $x_{0}$ only the state with lowest energy, $E$, survives. This state corresponds to the ground state of the associated real time Dirac equation

$$
\gamma_{0}^{M} E \psi=\left(\gamma_{\perp}^{M} p_{\perp}+\gamma_{3}^{M}(2 n+1) \pi T\right) \psi
$$

where the superscript, $M$, now indicates Minkowski $\gamma$-matrices. This equation is equivalent to a Dirac equation in $(2+1)$-dimensions with a "chiral" mass of $m=(2 n+1) \pi T$. This can be rotated into a real mass by the unitary transformation

$$
\psi \rightarrow e^{-i \frac{\pi}{4} \gamma_{3}} \psi
$$

Thus, we have

$$
\gamma_{0}^{M} E \psi=\left(\gamma_{\perp}^{M} p_{\perp}+(2 n+1) \pi T\right) \psi
$$

So far we have discussed the case of a massless particle. For massive fermions, one should use an effective mass of

$$
m_{\mathrm{eff}}=\sqrt{m_{d y n}^{2}+[(2 n+1) \pi T]^{2}} .
$$

Since lattice gauge calculations provide no conclusive evidence of a dynamical mass, we will restrict ourselves to the case of vanishing dynamical mass.

It is clear from eqn.(13) that the effective mass of the electron is very large at high temperature. The dynamics thus become nonrelativistic and can be described by a Schrödinger equation in two dimensions:

$$
E \psi=\frac{p_{\perp}^{2}}{2 m} \psi
$$


The effects of any additional potential (e.g., an "electrostatic" potential, $V=-e \Phi)$ can then simply be included by making the substitution $E \rightarrow E-V$ in eqn.(14).

These manipulations are readily extended to the present example of an interacting $e^{+} e^{-}$pair. (The details are given in the Appendix.) We note that the restriction to zero Matsubara frequency, $n=0$, in eqn.(35) represents a decision and not an approximation. It is also desirable to make the approximation of neglecting all terms, $\psi_{k}^{n}$, in eqn.(35) with $k \neq 0$. As we shall indicate shortly, this approximation introduces a tolerable relative error of $\mathcal{O}\left(e^{2}\right)$ for large $T$.

Before turning to numerical results, it is useful to perform a dimensional analysis aimed at understanding the high $T$ behavior of this system. For a wave function of size, $R$, the kinetic energy is equal to $\left(\pi T R^{2}\right)^{-1}$. Neglecting the binding energy, $E$, we obtain

$$
\frac{1}{\pi T R^{2}} \simeq e^{2} T
$$

Thus, the size of the bound system and the binding energy are

$$
R \sim \frac{1}{e T} \quad \text { and } \quad E \sim e^{2} T
$$

The size of the system is identical to electric screening length in a hot plasma which is also $(e T)^{-1} \cdot[2]$

With the approximations indicated above, the reduced mass for the $e^{+} e^{-}$pair becomes $m=\pi T / 2$, and the Schrödinger equation, eqn.(35), assumes the form

$$
-\frac{1}{\pi T}\left(\frac{1}{r} \frac{d}{d r}\left(r \frac{d \psi}{d r}\right)+l^{2} \psi\right)+V_{e f f}(r) \psi=E \psi .
$$

Using dimensionless units,

$$
x=\frac{e r}{\beta},
$$


we obtain for the $l=0$ state

$$
-\left(\frac{1}{x} \frac{d}{d x}\left(x \frac{d \psi}{d x}\right)\right)+\tilde{V}_{e f f}(x) \psi=\tilde{E} \psi
$$

where

$$
\tilde{E}=\frac{\pi E}{e T}+2 \pi \ln (2 T a)
$$

and

$$
\tilde{V}_{e f f}(x)=2 \pi \ln (x) .
$$

Note that eqn.(19) no longer depends on the physical parameters. Here, we have used the high temperature limit of the effective potential given by eqn.(4). The wave function is now given in terms of the dimensionless variable, $x$, rather than the physical parameters, $e$ and $T$. We have solved eqn.(19) numerically and the resulting wave function is plotted together with the potential in figure 2. Restoring dimensions, the energy is

$$
E=e^{2} T(1.45-2 \ln (e))
$$

We now consider a variety of corrections to these high- $T$ results which, in the approximation of zero dynamical mass, can be expressed purely in powers of our dimensionless parameter, the electric charge $e$. In particular, we consider (i) the non-asymptotic part of the potential, (ii) excitation of fermion modes with higher relative Matsubara frequencies $(k \neq 1)$, and (iii) relativistic corrections.

In figure 3 we compare the modified Coulomb potential in the periodic box given by eqn.(3) with its high- $T$, two-dimensional limit for a few values of the temperature. One can see that, although agreement is generally good enough, there are significant deviations 
at small distances: The original Coulomb potential, $e^{2} / 4 \pi r$, is still present in the original form. Since a typical separation is $r \sim 1 / e T$, this produces corrections to the energy of the system of $\mathcal{O}\left(e^{3} T\right)$.

The approximation of neglecting all terms in eqn.(35) with $k \neq 0$ can be relaxed perturbatively. Applying standard second-order perturbation theory one obtains

$$
\delta E=-\sum_{k} \frac{<1|V| k>^{2}}{E_{k}-E_{1}} \sim e^{4} T .
$$

Estimating the matrix elements as $V \sim \mathcal{O}\left(e^{2} T\right)$ and all energy denominators as $\mathcal{O}(T)$, one finds this correction to be $\delta E \sim e^{4} T$. As expected, this correction is smaller than the leading contribution to the energy, eqn.(25), by a factor of $\mathcal{O}\left(e^{2}\right)$. Taking only the first excited $(k=1)$ state into account numerical integration has produced the following result for $e^{2}=0.2$ :

$$
\frac{\delta E}{E}=3.5 \times 10^{-4}
$$

A typical velocity, $v_{t}$, transverse to the compact $z$-axis is $p_{t} / m_{\mathrm{eff}} \sim 1 / R T \sim e$. (The somewhat surprising feature of this result is that it is not $e^{2}$ as it would be for the $d=3$ hydrogen atom.) Therefore, relativistic corrections start at the level $E_{\text {rel }} \sim v_{t}^{2} E_{\text {non-rel }} \sim$ $e^{4} T$. A relativistic expansion similar to that leading to the Fermi-Breit Hamiltonian can be performed as in the $d=3$ case. We again have $-p^{4} / 8 m^{2}$ kinetic energy corrections, the spin-orbit term (which does not affect the ground state), spin-spin and "Darwin" terms.

We shall be concerned only with the properties of the spin-spin interaction since we wish to discuss the spin splitting in QCD in some detail in the next section. In the $d=2$ space one has symmetry only with respect to rotation in the $x y$-plane. Hence, states 
should be characterized by the $z$-component of their spin. In the ground state (with vanishing $z$-component of orbital angular momentum), the $e^{+} e^{-}$pair has two degenerate states with total spin $S_{z}= \pm 1$ and two distinct states with $S_{z}=0$ of different symmetry representing total spin $S=1$ and $S=0$ (ortho and para-positronium, respectively).

We start with with the textbook $(d=3)$ expression for the Fermi-Breit interaction between two opposite charges

$$
V_{\text {Breit }}=\frac{e^{2}}{4 m^{2}}\left[-\frac{\vec{\sigma}_{1} \cdot \vec{\sigma}_{2}}{R^{3}}+\frac{3\left(\vec{\sigma}_{1} \cdot \vec{R}\right)\left(\vec{\sigma}_{2} \cdot \vec{R}\right)}{R^{5}}+\frac{8 \pi}{3} \vec{\sigma}_{1} \cdot \vec{\sigma}_{2} \delta(\vec{R})\right]
$$

which consists of the familiar "tensor force" between magnetic moments and a contact interaction. In addition, in the case of positronium, one has to include an "annihilation" contribution

$$
V_{a n n}=\frac{\pi e^{2}}{2 m^{2}}\left(3+\vec{\sigma}_{1} \cdot \vec{\sigma}_{2}\right) \delta(\vec{R})
$$

Starting from these formulae, we can obtain the $d=2$ effective interaction (needed in the high $T$ limit) by averaging these potentials over $z$-direction. In three dimensions the tensor force vanishes for the spherically symmetric ground state. However, for finite temperature, the ground state has a "pancake" shape and a non-zero quadrupole moment. This leads to a contribution from the tensor force. This can be seen by rewriting eqn.(25) in momentum space as

$$
V_{\text {Breit }}=\frac{4 \pi e^{2}}{4 m^{2}} \int \frac{d^{3} q}{(2 \pi)^{3}} e^{i \vec{q} \cdot \vec{R}}\left[\vec{\sigma}_{1} \cdot \vec{\sigma}_{2}-\frac{\left(\vec{\sigma}_{1} \cdot \vec{q}\right)\left(\vec{\sigma}_{2} \cdot \vec{q}\right)}{q^{2}}\right] .
$$

Averaging over $z$ means that we select the component of the potential which has no momentum transfer in the $z$-direction. Therefore, the second term contributes only if the

\footnotetext{
${ }^{3}$ In the next section (in which we discuss the quark-antiquark interaction), the annihilation term is not present.
} 
spins are in the $x y$-plane:由

$$
\begin{aligned}
T \int d z V_{\text {Breit }} & =\frac{e^{2} T}{4 m^{2}} \int \frac{d^{2} q_{t}}{(2 \pi)^{2}} e^{i \overrightarrow{q_{t}} \cdot \vec{R}}\left[\vec{\sigma}_{1} \cdot \vec{\sigma}_{2}-\frac{\left(\vec{\sigma}_{1} \cdot \vec{q}_{t}\right)\left(\vec{\sigma}_{2} \cdot \vec{q}_{t}\right)}{q_{t}^{2}}\right] \\
& =\frac{e^{2} T}{4 m^{2}} 4 \pi \delta^{2}\left(R_{t}\right)\left[\vec{\sigma}_{1} \cdot \vec{\sigma}_{2}-\frac{1}{2} \vec{\sigma}_{1 t} \cdot \vec{\sigma}_{2 t}\right]
\end{aligned}
$$

\footnotetext{
${ }^{4}$ It is useful to consider an alternate derivation of the spin-spin interaction which offers a different explanation for why spins in the $z$-direction represent a special case. As for the Coulomb field in the box, one can satisfy periodic boundary conditions for the magnetic field by using a periodic array of reflected magnetic moments and by then noting that the $z$-directed moments display a strong tendency to cancel. A nice way of explaining this was suggested to us by H.A. Bethe. The Coulomb potential is a coherent sum of all reflections as they are all of the same sign. At high $T$, the potential of a charged wire results. Magnetic moments, on the other hand, can be considered as a pair of magnetic monopoles sitting on this wire with alternating signs. From this it is clear that the magnetic interaction vanishes since, for large $T$, the distance between these monopoles decreases.
} 
The remaining local terms simply lead to two-dimensional $\delta$-functions.

$$
\frac{1}{\beta} \int_{0}^{\beta} d z \delta^{3}(\vec{r})=T \delta^{2}\left(\vec{r}_{t}\right)
$$

As a result, the spin splitting of $d=2$ positronium is

$$
E_{\text {spin-spin }}=\frac{4 \pi e^{2} T|\psi(0)|^{2}}{m^{2}} C_{n}
$$

with the spin factors $C_{n}=3 / 4,1 / 2,-1 / 2$ for $S=1, S_{z}= \pm 1, S=1, S_{z}=0$ and $S=0, S_{z}=0$ states, respectively. 


\section{3. $\bar{q}$ "binding" in hot QCD}

As noted in the introduction, there exists a significant general difference between QCD and QED. Even at arbitrarily high temperature, one is confronted with non-trivial manifestations of the non-abelian nature of the former theory as one can see from the "dimensional reduction" argument [18]. The resulting $d=2+1$ theory is far from simple. In particular, as argued in 21, 22, it is known to produce a linear confining potential just as $d=1+1$ and $d=3+1$ (real QCD) theories do. In QED the electron charge is a physical parameter, and this can be assumed to be small in order to make the perturbative theory meaningful. In QCD we have a "running" coupling constant, and the actual magnitude of $\alpha_{s}=g^{2} / 4 \pi$ depends on the distances (or momenta) relevant for a given application. The precise value can only be specified if higher-loop corrections are calculated. In the high- $T$ limit, for example, we have a large effective mass and therefore the size of the Coulomb bound state is small, $R \sim \mathcal{O}(1 / g T)$. However, the logarithmic corrections are actually cut off at the smaller scale of $1 / T$. Therefore, (in contrast to quarkonium) one should expect that the relevant $\alpha_{s}$ is $\alpha_{s}(T)$ which becomes small as $T \rightarrow \infty$.

Unfortunately, the lattice data which we wish to discuss do not correspond to this relatively clean limit because they have been obtained at temperatures close to the critical temperature (i.e., $T \simeq 1.5 T_{c}$ ) which corresponds to effective masses of $m_{\mathrm{eff}}=\pi T \sim$ $450-600 \mathrm{MeV}$. From the experience of $J / \psi$ and $\Upsilon$ physics, we know that these masses are not sufficiently large to justify use of the Coulomb force alone. However, we have also learned another lesson from these examples: A non-relativistic approach based on a more

\footnotetext{
${ }^{5}$ This is similar to the case of superheavy quarkonium [23] with the obvious substitution $e^{2} \rightarrow$ $(4 / 3)\left(g^{2} / 4 \pi\right)$.
} 
complicated effective potential (which includes confining forces) works well in describing quarkonium bound states. It is reasonable to try the same approach in our "not-sohigh" $T$ case. In practice this means that, as in the previous section, we will rotate into the 'funny space' and solve a Schrödinger equation for quarks of mass $m_{\mathrm{eff}}=\pi T$. Consequently, the effective potential must also be given in this rotated space. Fortunately, such an interaction has been extracted on the lattice at high $T$ for pure glue [20]. The results are shown in figure 4 . Manosakis and Polonyi fit their results using the standard parameterization

$$
V=-a / r+\sigma r+\text { constant }
$$

with $a=0.184 \pm 0.02$ and $\sqrt{\sigma}=0.22 \pm 0.03$. These results can be compared with the values $a=0.26$ and $\sqrt{\sigma}=0.22 \pm 0.02$ obtained for $T=0$. If one identifies $\sigma$ with the phenomenological value of the string tension, $(400 \mathrm{MeV})^{2}$, the physical size of the lattice spacing is $a \sim 1 /(2 \mathrm{GeV}) \sim 0.1$ fm and $T \sim(1 / 3) \mathrm{GeV}$. The constant in eqn.(31) was not evaluated in ref.[20]. It is also instructive to compare these data with the potential appropriate for quarks propagating in the time direction which is also shown in figure 4 . Not only are the confining forces absent in this case (because we are well above the deconfinement temperature), but the Coulomb potential is much smaller due to screening effects. In contrast to these results, the potential measured in the spatial direction appears to be essentially $T$-independent, apart from some change at small distances. (This difference may simply result from the decrease of the effective Coulomb charge due to a difference in radiative corrections as $g(r) \rightarrow g(\beta)$.)

At this point one may ask how much of the LGT potential is simply due to the modified 
Coulomb potential obtained in the previous section,

$$
V=a(-1 / r+2 T \log (r / \beta))
$$

which results from the inclusion of "reflections" of the charge in the box. Fixing the coupling constant at small $\mathrm{r}$, one obtains the curve plotted in figure 4 which is not excluded by the LGT data. Thus, the data of ref. [20], do not rule out the possibility that the "spatial string tension" is actually very small. However, at the lower temperature, $T \simeq 210$ $\mathrm{MeV}$, where the 'wave functions' are being measured, the difference between the modified Coulomb potential and the string potential are substantial (under the assumption that the string tension remains the same). Therefore, by studying the wave function one can discriminate between these two alternatives. As we will show in the following, the 'wave functions' for $\pi$ and $\rho$ given in ref. [17] seem to require the presence of a string potential of the above magnitude.

With the potential fixed, one can proceed further and solve the $d=2$ Schrödinger equation numerically. In figure 5 we show the corresponding wave functions for several temperatures for both potentials eqn.(31) and eqn.(32). These results are compared to data at $T=210 \mathrm{MeV}$ These results suggest that the confining effective potential, eqn.(31), reproduces the data well while the modified Coulomb potential is definitely too weak at large distances 7 .

We now consider the role of spin-dependent forces. Comparing data for the $\rho$ and $\pi$ channels (shown in figure 5), one notes significant differences. The average size of the pion

\footnotetext{
${ }^{6}$ The data are taken at $\beta=6 / g^{2}=5.445$ with four time slices. Using the same $\beta$ and quark mass but six time slices, the authors derive a temperature of $T_{6}=140 \mathrm{MeV}$ [24]. This leads to a temperature of $T_{4}=210 \mathrm{MeV}$ for four time slices.

${ }^{7}$ Provided the coupling constants for the logarithmic and $1 / r$ parts of the potential are the same. Indeed, in this case, the standard 'asymptotic freedom' arguments do not apply. Logarithmic corrections are cut off at a scale of $1 / T$, so both terms should have the same coefficient, $\alpha_{s}(T)$.
} 
is significantly smaller than that of the $\rho$. At small distances $(r \leq 2 a)$, the pion wave function is much larger ${ }^{8}$ with $|\psi(0)|^{2}$ being about four times greater than in the $\rho$-meson case.

It is natural to ask whether this relative compactness of the pionic $q \bar{q}$-wave function results chiefly from the perturbative spin-spin interaction due to the exchange of high-frequency magnetic fluctuations. The corresponding ( $z$-averaged) interaction was discussed at the end of the previous section, and it can simply be used after (i) switching from standard QED to QCD units, $e^{2} \rightarrow(4 / 3)\left(g^{2} / 4 \pi\right)$, and (ii) ignoring the annihilation contribution in the case of these isospin 1 channels. Because the effects of this spin-spin interaction are not small, we cannot simply estimate them using perturbation theory. Rather, we must re-solve our effective Schrödinger equation including this spindependent, local interaction?.

Two sets of results at temperatures of 210 and $350 \mathrm{MeV}$ are shown in figure 6(a) and (b). The three curves correspond to $\left(S=1, S_{z}= \pm 1\right),\left(S=1, S_{z}=0\right)$ and $\left(S=0, S_{z}=0\right)$ states. A value of the effective coupling constant of $\alpha_{s}=g^{2} / 4 \pi=0.20$ was required to describe the difference between $\pi$ and $\rho$ wave functions found in LGT calculations. This value of $\alpha_{s}$ is about $50 \%$ larger than that used in the central interaction, eqn.(31). This difference may have several origins, but should not taken too seriously because, at

\footnotetext{
${ }^{8}$ It is worth noting that the $T=0$ pion wave function measured by Bernard et al. [17] is in principle the same as the zero temperature Bethe-Salpeter amplitude calculated by Chu, Lissia and Negele 25]. Both drop with an initial $e$-folding scale of $\sim 0.48 \mathrm{fm}$ which would give the pion an rms-radius of $\sim 1 / 3$ $\mathrm{fm}$. This is substantially smaller than the charge radius obtained in lattice gauge calculations of the density-density correlation function [26]. In terms of phenomenology [27, the Bethe-Salpeter amplitude provides the "intrinsic size" of the pion (probing its $\bar{q} q$-component) while most of the charge radius actually comes from the coupling of a virtual photon to the $\rho$-meson "cloud".

${ }^{9}$ In order to avoid difficulties arising from a zero-range potential, we represent the $\delta$-function appearing in $V_{\text {Breit }}$ by $\delta(x)=\sin (\Lambda x) / \pi x$. Here, $\Lambda$ represents a high frequency cutoff which we have chosen to be $\Lambda=\pi / a$ in order to simulate the effects of a finite lattice spacing, $a$.
} 
temperatures of $T \sim 210 \mathrm{MeV}$, the wave functions are rather insensitive to the strength of the central Coulomb potential एण. We remark that the lattice wave functions are not normalized. In figure 6(a) the two different states of the $\rho$ have the correct relative normalization, while the overall normalization of $\pi$ and $\rho$-wave functions is arbitrary. In figure 6(b) all three wave functions have the same normalization.

Our final point concerns the splitting of the screening masses. From the calculation displayed in figure 6, we obtain

$$
E_{\rho}-E_{\pi}=340 \mathrm{MeV}
$$

which can be compared with the results of direct measurements on the lattice with six time slices (i.e., at the lower temperature $(T=140 \mathrm{MeV})$, which gives $\sim 440 \mathrm{MeV}$.[24]

These predictions can be tested much more accurately by looking at the difference between the longitudinally and transversely $\left(S_{z}= \pm 1,0\right)$ polarized $\rho$ or by going to higher temperatures where our $d=2$ approximations should be better justified. Although the differences between wave functions for the different states of the $\rho$ shown in figure 6 appears to be rather small for both temperatures, their ratio (particularly at short distances) should provide sufficient information and should be less affected by systematic errors. The screening mass of these states differ by $\Delta M=0.25 T$

The general question of the physical origin of spin-dependent splittings in hadronic physics has been much debated in the literature. Together with the Breit-type perturbative interactions, interactions due to instantons, as proposed in refs. 30, 31, also produce a quasi-local interaction of the form $\left(\vec{\sigma}_{1} \cdot \vec{\sigma}_{2}\right)\left(t_{1}^{a} \cdot t_{2}^{a}\right)$. One way to distinguish them is to consider their temperature dependence, because the instanton density is expected to de-

\footnotetext{
${ }^{10}$ We point out that at $T=T_{c}$ Karsch [28] gives a value of $\alpha_{s}\left(T_{c}\right)=0.29$ for pure glue. Also, the $T=0$ value of $\alpha_{s}=0.3$ extracted from charmonium decay is of the same magnitude. [29].
} 
crease rapidly at high $\mathrm{T}$, while the perturbative mechanism can even be enhanced as the bound states discussed above become more compact.

Finally we mention that the effect of the Coulomb piece of the interaction, eqn.(31), on the wave function is tiny at those temperatures where the lattice data have been obtained. Therefore, it seems that these wave functions require the presence of a string-like confining potential. 


\section{Conclusion and Discussion}

Before summarizing our results, let us discuss the physical meaning of the spatial correlation functions. It has been already mentioned instead of yielding the more familiar spectrum of excitation energies, spatial correlators provide only the spectrum of momenta (or screening masses). In distinction to the situation at zero temperature, where these spectra are identical, the energy and momentum spectra are by no means similar at finite temperaturem. Unlike the former, momentum spectra display a very smooth $T$ dependence. There are two reasons for this fact. First, the quark effective mass consists of two parts, $m_{\mathrm{eff}}=\sqrt{\pi^{2} T^{2}+m_{d y n}^{2}}$. The first increases with $T$ and the second decreases as $T \rightarrow T_{c}$ with the result that the total value is roughly constant below $T_{c}$. Second, due to some dynamical reason (as yet unclear), the effective potential for spatial propagation is also temperature independent ${ }^{\mathrm{W}}$. As a result, there is a correspondence between the poles (and the corresponding "wave functions") for the $T=0$ and high- $T$ cases. In this (but only in this) sense, hadronic degrees of freedom smoothly traverse this boundary.

It is also worth recalling that the trend seen in the momentum spectra discussed above is quite different from what is known about the trends of the energy spectra. Various theoretical models suggest that the energy gaps (usually still referred to as "hadronic masses") decrease with T. (See, e.g., recent calculations based on hadronic scattering data [32].) There are suggestions that they even vanish at $T=T_{c}$ [15] so that, at higher

\footnotetext{
${ }^{11}$ We mention one more simple example which emphasizes the difference between the two. Consider non-interacting, massless fermions at high $T$. The excitation energy spectrum obviously starts from zero and corresponds to the cut while the momentum spectrum studied is a sequence of poles at $(2 n+1) \pi T$. The lowest pole is our "quark effective mass" in the 'funny space'.

${ }^{12}$ Apparently, this potential "does not notice" even a very strong first-order deconfinement transition which is observed in pure gluonic theory. Notice that this potential has not yet been evaluated when dynamical quarks are present.
} 
temperatures, the energy spectrum is just a cut containing excitations of any energies starting from zero. There is no contradiction between these two pictures: Propagation in space and in time at high $T$ are no longer simply related. Each can tell us something interesting about the properties of the QGP.

In this paper we have addressed not only these momentum (or "screening mass") spectra but also the corresponding wave functions. We have shown that the spatial correlation functions at high temperatures can be quite well understood in a 'funny space' obtained by interchanging time and space axes. The two main ingredients of our calculations are the quark effective mass, $m_{e f f}=\pi T$, and the effective potential, $V_{\text {eff }}$, which is a combination of a Coulomb and a confining part and is supplemented by a short-range spin-spin interaction. We find these ingredients to provide a good description of the wave functions measured in [17]. We further find that, at the temperatures for which lattice wave functions have been measured ( $T \simeq 210 \mathrm{MeV}$ ), the effect of the Coulomb piece of the effective interaction is small. Hence, we conclude that the lattice data require a relatively strong potential - much stronger than the modified Coulomb potential which would arise from dimensional reduction arguments [19]. Thus, as expected, dimensional reduction is not valid at temperatures as low as $T \simeq 210 \mathrm{MeV}$. Additional lattice calculations at considerably higher temperatures would be needed in order to check the validity of dimensional reduction.

The issue certainly can be pursued further both numerically and analytically. In particular, better data and data at higher temperatures are needed for further tests of the theory, as well as more detailed studies of the effective potential by itself a la 20]. For example, calculations of the effective potential with dynamical quarks are needed. Obviously, further attempts can be made in order to get a smooth matching between the 
traditional potential model description of hadrons at $T=0$ and our approach at high $T$. This could be done by solving the complete three dimensional Dirac equation with anti-periodic boundary conditions. Splittings between different spin-isospin components can be studied in greater detail.

What have we actually learned from these "wave functions" in simple physical terms? Consider a spatial correlation function at distance, $z$, with the two ends taken for simplicity at the same instant of time, and let us describe qualitatively the typical paths contributing to it. (This language is convenient because it is the same in physical and 'funny' space.) At $T=0$ the light quarks may travel by any path[ deviating from the $z$ axis by $\delta t \sim \delta x \sim \delta y \sim z$ (see figure ](a)) which leads to power-like correlation functions. At high $T$, for non-interacting quarks, the deviations in time are smaller, $\delta t \sim 1 / T$, due to the cancellation effects described in the introduction, and the correlator is $\exp (-M z)$. As a result, $\delta x \sim \delta y \sim \sqrt{z / T}$. (See figure ש(b).) These are the paths of "heavy" noninteracting quarks in the 'funny space'. Finally, for high $T$ and interacting quarks, quark and antiquark now travel along paths which are correlated within a distance governed by the "wave functions" considered above. (See figure 6(c).) Their deviation from the straight line remains as in case (b).

The perturbative Coulomb interaction in 'funny space' arises physically from the perturbative magnetic interaction of two color currents in original physical space. In the very high $T$ limit, when this effect dominates, the distance between them is $\delta x \sim \delta \sim 1 /(g T)$. Note that in a quark-gluon plasma the magnetic interaction is screened (if at all) only on a parametrically larger scale of $R_{m} \sim 1 /\left(g^{2} T\right)$. Thus, there is no contradiction here.

\footnotetext{
13 As usual, one can say that the correlation is caused by pair production somewhere in the middle, and its existence does not contradict causality because one cannot use it for signal transfer.
} 
Of course, the origin of the non-perturbative "confining" potential remains unclear. It is, in any event, due to some non-perturbative $G_{x y}$ field which is the magnetic field both in physical and 'funny' spaces.

It is important to distinguish between magnetic and electric interactions which are very different even in the more familiar electromagnetic plasma. The electric fields are screened and appear only as high frequency fluctuations. Magnetic fields are not, and they can produce very spectacular structures (as in the sun). In QCD the additional non-trivial feature is that the long-range magnetic fields are self-interacting, so their structure at high $\mathrm{T}$ is not understood at all.

As a final remark, let us try to answer the following question: Are the phenomena considered here subject to experimental observation? Correlations of electromagnetic currents are the source of photon and dilepton production from the plasma. Therefore, correlators similar to those introduced here will be essential in determining the effects of perturbative and non-perturbative gluomagnetic fields on these reactions. Such correlators are not easily obtained from LTG calculations. Here we have shown that a simple dynamical model is capable of reproducing the results of these more complicated LGT calculations for zero frequency correlators. This calculations have established a consistency between the zero frequency correlator and the spatial potential of ref.[20]. Moreover, they reveal the absolute necessity of a linearly rising piece in this potential. Given the success of this dynamical model and its relative ease of implementation, it seems both practical and promising to use it for more detailed calculations of photon and dilepton production. Thus, although much additional work needs to be done, it may turn out that the topic of this paper is not as academic as it may appear at first sight. 


\section{Acknowledgements}

We are particularly grateful to Carleton DeTar for communications and helpful advice. He also provided us with the data file for the lattice wave functions. This work was initiated by discussions with Tom DeGrand at Quark-Matter-91 where he reported the data under consideration. Discussions with I. Zahed at the initial stage of this work was also very important. Finally, we want to acknowledge fruitful discussions with H.A. Bethe on the spin-spin interaction. 


\section{Appendix}

In this appendix we will describe the details of the Schrödinger equation for an $e^{+} e^{-}$ pair. The wave function is characterized by the Matsubara frequency of the pair in 'funny space', $p_{z}=2 n \pi T$. The frequencies of the electron and positron must sum to this value but their difference is free. The wave function for the $e^{+} e^{-}$pair can thus written as

$$
\Psi^{n}=\sum_{k=-\infty}^{\infty} c_{k} \psi_{k}^{n}
$$

where $n$ is the sum of the Matsubara frequencies and $-\infty \leq k \leq+\infty$ their difference. The coefficients, $c_{k}$, are determined from the solution of the following Schrödinger equation:

$$
\begin{aligned}
& \left(\pi T(|n+k+1|+|n-k-1|)+\frac{p_{\perp, 1}^{2}}{2(n+k+1) \pi T}+\right. \\
& \left.\quad \frac{p_{\perp, 2}^{2}}{2(n-k-1) \pi T}+V_{0}\right) \psi_{k}^{n}+\sum_{l=+1}^{+\infty} V_{2 l}\left[\psi_{k-l}^{n}+\psi_{k+l}^{n}\right]=E^{n} \psi_{k}^{n}
\end{aligned}
$$

where $V_{o}$ is the effective Coulomb Potential (eqn.(3)) and

$$
V_{2 l}\left(\vec{r}_{\perp}\right)=\frac{T}{(2 \pi)^{2}} \int d^{2} k_{\perp} e^{i \vec{r}_{\perp} \cdot \vec{k}_{\perp}} \frac{1}{k_{\perp}^{2}+(2 l \pi T)^{2}}
$$

are the higher Matsubara components of eqn.(2).

These energies and wave functions are the immediate building blocks for the various correlators which we can construct. In the original, unrotated space, these are given by

$$
\begin{aligned}
C\left(\vec{r}_{\perp}, z, \tau\right) & =\int d^{2} x_{\perp}<\hat{T}\left[\hat{\mathcal{O}}\left(\vec{x}_{\perp}, z=0, \tau=0\right) \bar{q}\left(\vec{x}_{\perp}, z, \tau\right) \Gamma q\left(\vec{x}_{\perp}+\vec{r}_{\perp}, z, \tau\right)\right]> \\
& =\sum_{n} \exp \left(-i \omega_{n} \tau\right) C\left(\vec{r}_{\perp}, z, \omega_{n}\right)
\end{aligned}
$$

where the "source" $\hat{\mathcal{O}}$ is an operator which creates quark and antiquark at $z, \tau=0, \Gamma$ the appropriate Dirac matrix for the meson under consideration and $\omega_{n}$ are the usual 
Matsubara frequencies. The Matsubara components of the above defined correlation functions are then related to the solution of the Schrödinger equation (35) by

$$
C\left(\vec{r}_{\perp}, z, \omega_{n}\right)=\sum_{\alpha} a_{\alpha} \exp \left(-M_{\alpha}^{n} z\right) \Psi_{\alpha}^{n}\left(\vec{r}_{\perp}\right)
$$

The index, $\alpha$, labels the eigenstates of eqn.(35), $M_{\alpha}^{n}$ are the associated screening masses of these states which are identical with the eigenvalues of the Schrödinger equation (35) and $a_{\alpha}$ are some coefficients which we do not further specify. In the limit of large $z$ only the ground state survives and we have

$$
\lim _{z \rightarrow \infty} C\left(\vec{r}_{\perp}, z, \omega_{n}\right) \propto \exp \left(-M_{0}^{n} z\right) \Psi_{0}^{n}\left(\vec{r}_{\perp}\right)
$$

where $\Psi_{0}^{n}$ denotes the solution of eqn.(35) of lowest energy for given $\mathrm{z}$-momentum in 'funny space' $p_{z}=2 \pi n T=\omega_{n}$.

The primary focus of LGT so far has been the zero frequency correlator (i.e., $n=$ 0). This is achieved by integrating the correlator, eqn.(37), over $\tau$. In addition, an uncorrelated source operator of the form

$$
\mathcal{O}_{\text {uncorr. }}\left(\vec{x}_{\perp}, z, \tau\right)=\int d \tau^{\prime} d^{2} x_{\perp}^{\prime} \bar{q}\left(\vec{x}_{\perp}, z, \tau\right) \Gamma q\left(\vec{x}_{\perp}+\vec{x}_{\perp}^{\prime}, z, \tau+\tau^{\prime}\right)
$$

is used. This source sends off a quark-antiquark pair with vanishing relative Matsubara frequency and transverse momentum.

Finally, notice that transverse wave functions result from correlations between quark and antiquark at equal time because both operators are defined at the same time, $\tau$, in eqn.(37). 


\section{References}

[1] D. J. Gross, R. D. Pisarski and L. G. Yaffe, Rev. Mod. Phys. 53, 43 (1981).

[2] E. Shuryak, Phys. Rep. 61, 72 (1980).

[3] C. DeTar, Phys. Rev. D 32, 276 (1985).

[4] C. DeTar, Phys. Rev. D 37, 2328 (1987).

[5] C. DeTar and J. Kogut, Phys. Rev. Lett. 59, 399 (1987).

[6] C. DeTar and J. Kogut, Phys. Rev. D 36, 2828 (1987).

[7] K. Born, S. Gupta, A. Irbäck, F. Karsch, E. Laermann, B. Petersson, and H. Satz (MT ${ }_{c}$ Collaboration), Phys. Rev. Lett. 67, 302 (1991).

[8] T. Hatsuda and T. Kunihiro, Phys. Lett. B 145, 7 (1984).

[9] T. Hatsuda and T. Kunihiro, Phys. Rev. Lett. 55, 158 (1985).

[10] T. Hatsuda and T. Kunihiro, Prog. Theor. Phys. 74, 765 (1985).

[11] A. Gocksch, Chiral symmetry in hot QCD, Brookhaven preprint BNL-46286,1991.

[12] V. Eletskii and B. Ioffe, Sov. J. Nucl. Phys 48, 602 (1988).

[13] E. Shuryak, Invited talk at Quark Matter-91, Gatlinburg, 1991, Nucl.Phys.A, in press.

[14] H. Bethe, G. Brown, A. Jackson, and P. Pizzochero, subm. to Nucl. Phys. A.

[15] G. Brown and M. Rho, Phys.Rev.Lett. 66, 2720 (1991). 
[16] G. Brown, H. Bethe, and P. Pizzochero, Phys. Lett. B 263, 337 (1991).

[17] C. Bernard, M.C. Ogilvie, T. DeGrand, C. DeTar, S. Gottlieb, A. Krasnitz, R. Sugar, and D. Toussaint, 'The spatial structure of screening propagators in hot QCD' University of Arizona report AZPH-TH/91-60, (1991)

[18] T. Appelquist and J. Carazzone, Phys. Rev. D 11, 2856 (1975).

[19] T.H. Hanson and I. Zahed, Stony Brook preprint SUNY-NTG-91-44.

[20] E. Manosakis and J. Polonyi, Phys. Rev. Lett. 58, 847 (1987).

[21] E. D’Hoker, Nucl.Phys.[FS4] B 200, 517 (1982).

[22] C. Borgs, Nucl.Phys. B 261, 455 (1985).

[23] T. Appelquist and H. D. Politzer, Phys. Rev. Lett. 34, 43 (1975).

[24] C. Bernard, M.C. Ogilvie, T. DeGrand, C. DeTar, S. Gottlieb, A. Krasnitz, R. Sugar, and D. Toussaint, 'QCD thermodynamics with two flavors at $N_{t}=6$ ' University of Arizona report AZPH-TH/91-45, (1991)

[25] M.C. Chu, M. Lissia, and J.W. Negele, Nucl. Phys. B 360, 31 (1991).

[26] M. Lissia et al, MIT CTP Preprint 1922 (1991).

[27] G.E. Brown, M. Rho and W. Weise, Nucl. Phys. A 454, 669 (1986) .

[28] F. Karsch, Z. Phys. C 38, 147 (1988).

[29] D. Coffman et al., Phys. Rev. Lett. 68, 282 (1992). 
[30] E. Shuryak, Nucl. Phys. B 214, 237 (1983).

[31] E. Shuryak and J. L. Rosner, Phys. Lett. B 218, 72 (1989).

[32] E. Shuryak, Nucl. Phys. A 533, 761 (1991). 


\section{Figure captions:}

Figure 1: Schematic picture of replications leading to the two-dimensional Coulomb potential (3)

Figure 2: Wave function (full line) and potential (dashed line) for two-dimensional positronium with $x=r(e T)$

Figure 3: Comparison of finite temperature Coulomb potentials. The solid lines correspond to the potential (3), while the dashed ones correspond to the two-dimensional potential, $2 T \log (r T)$. 
Figure 4: The potential of ref. [20]. The finite temperature $\left(T>T_{c}\right)$ space-like potential corresponds to the full line, the zero temperature time-like potential to the dashed line. The dashed-dotted line represents the finite temperature $\left(T>T_{c}\right)$ space-like potential and the short-dashed-long-dashed line the modified Coulomb potential according to eqn.(3).

Figure 5: Wave function for the $\rho$-meson as function of $\mathrm{r} / \mathrm{a}(a=0.22 \mathrm{fm})$ for different temperatures. The upper set of curves corresponds to wave functions obtained with the modified Coulomb potential (eqn.(32)) the lower set with the confining potential (eqn.(31)). Temperatures: $150 \mathrm{MeV}$ (full line), $250 \mathrm{MeV}$ (short-dashed line) and $350 \mathrm{MeV}$ (long-dashed line). Data are form ref. [17] and correspond to $T \simeq 210 \mathrm{MeV}$.

Figure 6: Wave functions for $\rho$ and $\pi$ at $T=210 \mathrm{MeV}$ (a) and $T=350 \mathrm{MeV}$ (b) as a function of $r / a(a=0.23 \mathrm{fm})$. The full line and the short-dashed line correspond to the $\rho$ with spin quantum number $S_{z}= \pm 1$ and $S_{z}=0$ respectively. The long-dashed line represents the pion wave function. In (a) arbitrary normalization was used while in (b) all wave functions have the same normalization. Data are form ref.[17].

Figure 7: Different paths contributing to a equal time correlator of two currents separated by a distance $z$. In the $T=0$ case (a) the transverse deviation of the paths is $r_{\perp} \sim z$ while at high $T$ for non-interacting quarks (b) it is smaller, $r_{\perp} \sim \sqrt{z / T}$. The bound quarks (c) still deviate from the axis by $r_{\perp} \sim \sqrt{z / T}$, but their relative distance is defined by the size of the bound state $r_{\text {bound }}$. 